population of LP-PWLH and compare the VS to the group who had earlier access (EA) to prenatal care.

Methods A retrospective cohort carried out at the major HIV reference centre in Bahia, Brazil. Medical records of PWLH attended at prenatal care were reviewed from January 2011 to December 2013. HIV VL and TCD4+ count data were obtained from the national database. Statistical analyses were performed with SPSS 20.0.

Results A total of 235 PWLH enrolled in the study, of which $29.4 \%$ were LP. Among the latter, the mean age was 28.3 $( \pm 6.9)$ years, similar to the EA group. Thirty four percent of the LP had $<8$ schooling years $(\mathrm{p}=0.16), 40.7 \%$ were single $(p=0.64), 24.6 \%$ reported alcohol use $(p=0.15), 1.6 \%$ drug use $(p=0.44)$ and only $16.7 \%$ regular condom use $(p=0.92)$. The majority of LP (62.9\%) had partners with unknown serological status, $25.7 \%$ had seroconcordant and $11.4 \%$ had serodiscordant partners $(\mathrm{p}<0.01)$. LP predominantly had HIV diagnosis during pregnancy $(60.9 \% ; \mathrm{p}<0.01)$ and were ARV naïve $(78.3 \% ; \mathrm{p}<0.01)$, while only $14.5 \%$ were on ART at conception $(\mathrm{p}<0.01)$. As for the initial ART regimen during pregnancy, $89.9 \%$ of LP were using a protease inhibitor based regimen and $11.6 \%$ had had regimen changes during pregnancy $(p=0.36)$. LP had a higher initial VL $\left(\log _{10} 3.4\right.$; $\mathrm{p}<0.01$ ) and those with recent diagnosis also had higher VL $\left(\log _{10} 3.8 ; \mathrm{p}=0.02\right)$. LP were more likely to not have a second VL during pregnancy or early peripartum $(33.3 \%$; $\mathrm{p}<0.01)$. VS was less achieved $(34.8 \%$ vs $71.8 \% ; \mathrm{p}<0.01$; OR 4.7, CI95\% 2.36-9.66) by the LP group.

Conclusion LP showed an increased risk of MTCT, with recent HIV diagnosis, higher VL at prenatal onset and a lower rate of VS. Thus, the use of integrase inhibitors would be a better choice for this population, since it promotes a quickly decrease of VL.

\section{P3.76 HTLV-1/-2 SEROLOGY IN THE TESTS BATTERY FOR FOLLOWING UP PATIENTS WITH VIRAL HEPATITIS IN BRAZIL}

${ }^{1} \mathrm{FA}$ Alves, ${ }^{1} \mathrm{KR}$ Campos, ${ }^{2} \mathrm{MF}$ Lemos, ${ }^{2} \mathrm{RC}$ Moreira, ${ }^{1} \mathrm{~A}$ Caterino de Araujo. ${ }^{1}$ Centro de Imunologia (Laboratório de HTLV); ${ }^{2}$ Centro de Virologia (Laboratório de Hepatites, Núcleo de Doenças de Transmissão Sanguínea e Sexual), Instituto Adolfo Lutz, São Paulo, SP., Brazil

10.1136/sextrans-2017-053264.311

Introduction Brazil is endemic for HIV-1, HTLV-1 and HTLV2 , these retrovirus share routes of transmission with $\mathrm{HCV}$ and $\mathrm{HBV}$, thus coinfections can occur. Several studies tried to evaluate the impact of human retroviruses on the course of HCV infection, and association of HTLV-1 with spontaneous clearance of HCV, mostly in HIV coinfected patients, and less hepatic injury were detected. In contrast, an increase in $\mathrm{HCV}$ viral load in HIV and/or HTLV-2 coinfected individuals was described. Concerning HBV infection, one study showed higher rate of $\mathrm{HBV}$ antigenemia in HIV/HTLV-1 coinfected patients. Thus, searches for HTLV infections in HCV and $\mathrm{HBV}$ infected patients have prognostic value.

Methods Plasma samples from 1244 individuals sent to Instituto Adolfo Lutz for measuring HCV and HBV viral load: $622 \mathrm{HCV}+(\mathrm{G} 1=343$ male, 279 female $)$, and $622 \mathrm{HBV}+$ (G2=327 male, 295 female) were evaluated for HTLV-1/-2 infection by enzyme immunoassay (EIA, HTLV-I/II, Gold ELISA, REM), and confirmed by line immunoassay (INNOLIA HTLV-I/II, Fujirebio). HIV infection was detected by immunochromatographic assay (Rapid Check HIV 1+2, UFES).

Results On screening test 44 plasma samples reacted, and HTLV-1 was confirmed in 25 samples [20(G1), 5(G2)]. HTLV2 was detected in 16 samples [13(G1), 3(G2)]. Two samples were indeterminate, and one negative (G2). The overall prevalence of HTLV in HCV+ was 5.3\% (3.2\% HTLV-1\% and $2.1 \%$ HTLV-2), and HBV $+1.3 \% \quad(0.8 \% \quad \mathrm{HTLV}-1 \%$ and $0.5 \%$ HTLV-2). No difference in the median age of patients was detected between HCV-infected and HCV/HTLV coinfected (50.7 vs. 50.6 years), also in HBV and HTLV/HBV coinfected (45.8 vs. 53.5 years). In HCV/HTLV coinfected patients $30.3 \%$ were HIV+, while in HBV/HTLV coinfected patients, all except one were HIV+.

Conclusion The results emphasise the need for searching HTLV infections mostly in patients with HCV. Thus, we suggest to include the serology for HTLV in the tests battery for following up the hepatitis virus infected patients in Brazil, regardless of your HIV status.

Support: FAPESP AP\#2016/03654-0, CNPq PD\#302661/ 2015-8

\section{P3.77 MOTHER-TO-CHILD TRANSMISSION OF HIV IN SOUTHERN SANTA CATARINA, BETWEEN 2005 AND 2015: ASSESSMENT OF SEROCONVERSION}

${ }^{1}$ Karen Waleska Kniphoff de Oliveira, 'Suzana Kniphoff de Oliveira, ${ }^{1}$ Ana Beatriz Sanches Barranco, ${ }^{1}$ Tamara Hoffmann, ${ }^{1}$ Camila Soares Duarte, ${ }^{1}$ Rayane Felippe Nazário, ${ }^{2}$ Chaiana Esmeraldino Mendes Marcon, ${ }^{2,3}$ Fabiana Schuelter-Trevisol. 'Medical School at University of Southern Santa Catarina, Brazil; ${ }^{2}$ Postgraduate Program in Health Sciences at University of Southern Santa Catarina, Brazil; ${ }^{3}$ Clinical Research Centre at Hospital Nossa Senhora da Conceição, Brazil

\subsection{6/sextrans-2017-053264.312}

Introduction In Brazil, 92,210 HIV-infected pregnant women were notified from 2000 through June 2015, most of whom living in the Southeast (40.5\%) and South (30.8\%). Detection rates of pregnant women living with HIV in Brazil have increased in the last ten years. In 2005, rates of seropositivity for newborns were as high as 2.0 cases per 1000 live births, which increased to 2.6 in 2014 , indicating a $30.0 \%$ rise. The aim of this study was to analyse the frequency of seroconversion among newborns to HIV-positive mothers living in southern Santa Catarina, Brazil, from 2005 through 2015.

Methods A cross-sectional study was conducted to collect secondary data. All the newborns that were exposed to HIV by vertical transmission, and attended the municipal healthcare centre between 2005-2015 participated in the study. The study included all infants between 0 and 18 months of age, exposed to HIV vertically, who attended the healthcare centre that serves 18 municipalities in southern Santa Catarina, Brazil, over the 2005-2015 period.

Results During the study period, there were 93 exposures to HIV, of which $3(3.2 \%)$ seroconversions were confirmed and $2(2.1 \%)$ died of AIDS during the follow-up period. Seroconversion was associated with breastfeeding $(\mathrm{PR}=29.3$; 95\% $\mathrm{CI}=9.6-89.2 ; \mathrm{p}=0.002)$ and the lack of antiretroviral therapy during pregnancy $(\mathrm{PR}=21.0 ; 95 \% \mathrm{CI}=2.4-184.5$; $\mathrm{p}=0.006$ ).

Conclusion The results from this study allowed us to conclude that seroconversion among newborns was 5.4\%, resulting in a rate of 3.4 cases per 1000 live births, which was higher than the national average. Seroconversion was associated with 